\title{
A review of virtual planning software for guided implant surgery - data import and visualization, drill guide design and manufacturing
}

Florian Kernen ${ }^{1 *}$ D, Jaap Kramer ${ }^{1}$, Laura Wanner ${ }^{1}$, Daniel Wismeijer ${ }^{2}$, Katja Nelson $^{1}$ and Tabea Flügge ${ }^{1,3}$

\begin{abstract}
Background: Virtual implant planning systems integrate (cone beam-) computed tomography data to assess bone quantity and virtual models for the design of the implant-retained prosthesis and drill guides. Five commercially available systems for virtual implant planning were examined regarding the modalities of integration of radiographic data, virtual dental models and the design of drill guides for guided implant surgery. The purpose of this review was to describe the limitations of these available systems regarding the import of imaging data and the design and fabrication of a drill guide.
\end{abstract}

Methods: The following software systems were examined regarding the import of imaging data and the export of the virtual implant planning for the design and fabrication of a drill guide with the help of two clinical situations requiring dental implant therapy: coDiagnostiX ${ }^{\mathrm{TM}}$, DentalWings, Canada (CDX); Simplant Pro ${ }^{\mathrm{TM}}$, Dentsply, Sweden (SIM); Smop ${ }^{\mathrm{TM}}$, Swissmeda, Switzerland (SMP); NobelClinician ${ }^{\mathrm{TM}}$, Nobel Biocare, Switzerland (NC); Implant Studio, 3Shape, Denmark (IST). Assessment criteria included data formats and management as well as the workflow for the design and production of drill guides.

Results: All systems have a DICOM-interface ("Digital Imaging and Communication in Medicine") for the import of radiographic data. Imaging artefacts could be reduced but not eliminated by manual data processing. The import of virtual dental models in a universal format (STL: Standard Tesselation Language) was possible with three systems; one system could only be used with a proprietary data format.

All systems display three-dimensional surface models or two-dimensional cross-sections with varying orientation for virtual implant planning. Computer aided design and manufacturing (CAD/CAM) of drill guides may be performed by the user with the help of default parameters or solely by the provider of the software and thus without the influence of the clinician.

\footnotetext{
* Correspondence: florian.kernen@uniklinik-freiburg.de

'Department of Oral and Maxillofacial Surgery, Translational Implantology, Medical Center - University of Freiburg, Faculty of Medicine, University of Freiburg, Hugstetter Str. 55, 79106 Freiburg, Germany

Full list of author information is available at the end of the article
}

C C The Author(s). 2020 Open Access This article is licensed under a Creative Commons Attribution 4.0 International License, which permits use, sharing, adaptation, distribution and reproduction in any medium or format, as long as you give appropriate credit to the original author(s) and the source, provide a link to the Creative Commons licence, and indicate if changes were made. The images or other third party material in this article are included in the article's Creative Commons licence, unless indicated otherwise in a credit line to the material. If material is not included in the article's Creative Commons licence and your intended use is not permitted by statutory regulation or exceeds the permitted use, you will need to obtain permission directly from the copyright holder. To view a copy of this licence, visit http://creativecommons.org/licenses/by/4.0/. The Creative Commons Public Domain Dedication waiver (http://creativecommons.org/publicdomain/zero/1.0/) applies to the data made available in this article, unless otherwise stated in a credit line to the data. 
(Continued from previous page)

Conclusion: Data bases of commonly used implant systems are available in all tested software, however not all systems allow to plan and execute fully guided implant placement. An individual design and in-house manufacturing of the drill guide is only available in some software systems. However, at the time of publication most recent software versions showed flexibility in individual design and in-house manufacturing of drill guides.

Keywords: Guided implant surgery, Computer-assisted surgery, Computer-aided design, Virtual implant planning

\section{Background}

Conventional implant planning is based on clinical examination and $2 \mathrm{D}$ radiographic imaging. The adoption of $3 \mathrm{D}$ radiographic imaging enables a more precise diagnosis of residual bone dimensions, the intrabony course of the inferior alveolar nerve and neighboring teeth $[1,2]$.

Individual patient 3D-imaging data is essential for virtual dental implant planning, computer aided design (CAD) and computer aided manufacturing (CAM) of a drill guide or implant-supported prosthesis. Anatomical data is derived from (cone beam) computed tomography (CT or CBCT) and optical scans of teeth and mucosa.

CBCT has a lower radiation dose $(92-118 \mu \mathrm{Sv})$ than CT $(860 \mu \mathrm{Sv})$ and is therefore often used for dental implant planning $[3,4]$. Both $\mathrm{CT}$ and $\mathrm{CBCT}$ are stored in the universal format for "Digital Imaging and Communication in Medicine" (DICOM-format). Amongst imaging data, geometric and mathematical information, practical information such as acquisition details and settings are included in the DICOM file.

Volumetric imaging data is displayed in 2D crosssectional images aligned to the prospective implant position. 3D surface models of CT or CBCT data are displayed using segmentation. Each voxel in the volumetric data set is assigned a grey value following its radiation attenuation, depending on the specific tissue characteristics. The display of a limited range of grey values enables the selective display of specific anatomical structures (segmentation).

CT or CBCT does not sufficiently display the tooth surface for the prosthetic set-up and for drill guide production. Especially in the presence of restorations, artifacts such as streaks and extinct areas occur [5]. Therefore, CT or $\mathrm{CBCT}$ scans and a virtual dental model obtained either from an intraoral optical scan or an extraoral scan of impressions or stone casts are aligned to each other prior to implant planning [6].

The data of intra- and extraoral optical scans are usually available in the universal stereolithography format (STL). This format contains geometric information of the surface [7]. Virtual dental models can be displayed in $2 \mathrm{D}$ along cross-sections and $3 \mathrm{D}$ to assess the mucosal surface from different viewpoints.

The process of aligning multiple imaging datasets with each other is defined as registration [8, 9]. Different procedures can be used to accomplish an accurate registration of CT or CBCT scans and virtual dental models: The tooth surface as a common structure displayed in both datasets may be used for registration. Custom and standardized reference markers (fiducial markers), respectively, can otherwise be introduced with a radiographic splint [10].

With standardized markers stored in the software, a single scan of the patient wearing the radiographic splint is performed (single scan protocol) $[11,12]$. In the software, the stored reference marker is registered with the scanned image of the respective marker.

With custom markers a double scan protocol is used: after CT or CBCT acquisition of the patient with the radiographic splint, the radiographic splint alone is scanned $[10,13,14]$. The images of reference markers in both datasets are registered.

When using the tooth surface as a reference for registration, a splint with fiducial markers is not necessary [6, $15,16]$. The software uses an algorithm to register corresponding anatomical surfaces (automatic registration) or requires previous selection of corresponding areas by the user to initiate the registration process (semi-automatic registration). The accurate registration of $\mathrm{CT}$ or $\mathrm{CBCT}$ data and virtual models is crucial for a precise transfer of the prospective implant position to the surgical site [9].

After data import, segmentation and registration the prosthetic set-up and virtual implant position is planned. The prosthetic set-up combines the ideal position of implant-supported prosthesis and takes the abutment design with its emergence profile, morphology of the tooth, occlusal and proximal contacts into consideration. Using this information, implants can be virtually positioned in cross-sectional images and three-dimensional surface models reconstructed from the radiographic volume.

The design of a drill guide can vary depending on its function. It can either a) only guide the pilot drill (pilot guided) or b) guide every drill of the implant specific drill sequence (fully guided) [15, 17]. Additional to fully guided drilling, implant placement can be performed through the drill guide [11]. Guided protocols are preferred to complete free handed drilling and implant placement due to a higher accuracy of the implant position [14]. 
Drill guides may either be supported by the remaining teeth, the mucosa, directly by the bone surrounding the implant or by temporarily inserted mini implants [18, 19]. Especially in edentulous jaws with a mucosal support, the stability may be ameliorated with transitional screws or pins or temporary implants, securing the drill guide to the bone [20,21].

In a fully digital workflow drill guides are virtually designed (CAD) and produced using computer-aided manufacturing (CAM). CAD/CAM is either performed by the software user or in a central production facility. The guides are milled from resin blanks [22, 23] or produced with an additive technique e.g. rapid prototyping [24]. In a combination of analog and digital techniques, drill guides are adapted from conventionally produced radiographic splints or produced on stone casts.

In this narrative review, the possibilities and limitations of five commercially available implant planning software systems are examined regarding the import of imaging data and the export of the virtual implant planning for the design and fabrication of a drill guide.

\section{Methods}

The following commercially available virtual implant planning systems: coDiagnostiX, Version 9.9. (DentalWings, Canada) (CDX); Simplant Pro, Version 17 (Dentsply, Sweden) (SIM); Smop, Version 2.13. (Swissmeda, Switzerland) (SMP); NobelClinician, Version 2.4. (Nobel Biocare, Switzerland) (NC); ImplantStudio, Version 1.6.4.4, (3Shape, Denmark) (IST) were examined.

\section{Study design}

The study design included the review of five different planning systems. Data of two patients with different indications for dental implant treatment were used to assess import and processing of imaging data for dental implant planning and drill guide production using CAD/CAM technology.

One patient had a missing single tooth in the region 21 (FDI), a fixed metal-ceramic prosthesis in the first quadrant and a cast post and core and metal-ceramic crown on the adjacent tooth (11 FDI). The second patient presented with a partially edentulous jaw with missing teeth in region 45-47 (FDI), a metal-ceramic crown on the adjacent tooth (44 FDI) and no other restorations in the lower jaw. CBCT data (3D Accuitomo, Scanora) and intraoral optical scans of the first patient (iTero, Cadent, Santa Clara, CA, US) as well as digitized stone casts (D250, 3Shape, Copenhagen, Denmark) of the second patient were available. CBCT data was stored in a DICOM format. Intraoral and extraoral scans and stone cast scans were available in the universal file format (STL). The above-mentioned virtual implant planning systems were evaluated by one examiner with defined assessment criteria as follows:

\section{Data acquisition and registration}

Each system was examined regarding its options for the import of radiographic data (CT or $\mathrm{CBCT}$ ) and virtual dental models. The availability of a proprietary scanner for intraoral scans or extraoral model and impression scans, respectively, and the data format specification for data import were assessed. Settings for the alignment of virtual dental models and radiographic data were evaluated regarding the use of single and double scan protocols and assistance of the system in the registration process (semi-automatic, automatic) (Table 1).

\section{Visualization of imaging data}

The visualization of CT or CBCT data was compared between the systems, regarding the options to select grey values for the display of distinct structures. Grey values for a segmented display of anatomy were selected manually or with pre-settings for certain structures (e.g. skin, bone, teeth). The selection of three-dimensional display options of CT or CBCT data, the availability of crosssections as well as their setting and the orientation of models with the help of standard planes and views were assessed (Table 2).

\section{CAD/CAM of drill guides}

The spatial coordinates of the planned implant position were used for CAD/CAM of the drill guide. The possibilities for its design were examined for each system. The provided tools for fit, support and material thickness were documented. The options of in-house (individual) or centralized production of the drill guides were assessed (Table 3).

\section{Results}

\section{Data acquisition and registration}

All examined systems used the universal DICOM format for $\mathrm{CT}$ or $\mathrm{CBCT}$ data import. The five examined systems allowed the import of scanning data in the universal STL-format (CDX, SIM, SMP, IST), while one system (IST) offered proprietary intraoral scanning and dental laboratory scanning technology (Trios, 3Shape). Intraoral scans acquired with Trios were displayed in Implant Studio $^{\mathrm{nt}}$ with the texture and color of the teeth and mucosa. Another software (CDX) was linked with the software Cares (Dentalwings, Montreal, Canada) that provided a

Table 1 Assessment criteria for data acquisition and registration of image data

\begin{tabular}{ll}
\hline $\begin{array}{l}\text { intraoral scans } \\
\text { extraoral scans }\end{array}$ & $\begin{array}{l}\text { importable data formats } \\
\text { proprietary scanner available } \\
\text { (CB-)CT }\end{array}$ \\
specifications (data format, image resolution) \\
image registration & $\begin{array}{l}\text { single scan protocol using reference markers } \\
\text { single scan protocol using tooth surface } \\
\text { double scan protocol }\end{array}$ \\
\hline
\end{tabular}


Table 2 Assessment criteria for visualization of imaging data visualization of dental models

visualization $\mathrm{CT}$ or $\mathrm{CBCT}$ data

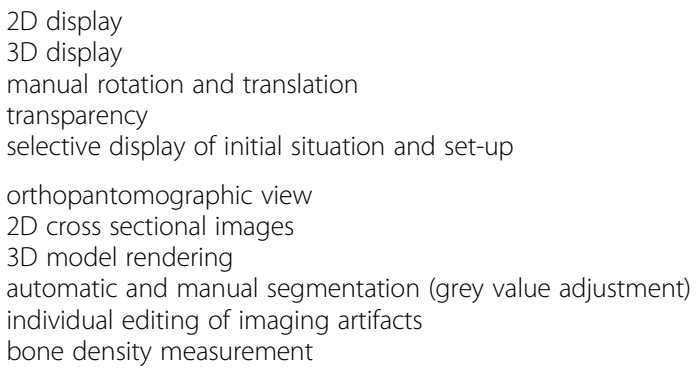

proprietary dental laboratory scanner for stone casts and dies. Both proprietary scanning systems provided virtual models as universal STL- files that might be used with any dental implant planning system (Fig. 1). One implant planning system (NC) exclusively imported model scans in a proprietary data format called NXA that could only be acquired with the proprietary dental laboratory scanner NobelProcera G2 (Nobel Biocare, Switzerland). The use of intraoral scans for implant planning was not possible with this system, as no proprietary intraoral scanner existed and no third-party intraoral scanner produced the NXA-data format.

The examined software systems offered different protocols for registration of CT or CBCT data and surface models. For single scans without reference markers, semiautomatic (CDX, SMP, SIM) or automatic (NC) registration algorithms using the tooth surface as a common structure in both images were applied. To initiate the semiautomatic registration process, the user selected corresponding areas on the surface segmented from CT or CBCT data and on the surface model of the teeth. In case of a visible deviation between the models, all examined software systems required the user to adjust the registration by manually moving the models in threedimensional space (Table 4).

\section{Visualization of imaging data}

All examined systems provided tools for the selection of grey values for a selective display of anatomical structures. Bone and teeth could be visualized by selecting the appropriate range of grey values according to their density (Fig. 1). Default grey values for bone, teeth or soft tissues were given in all systems (CDX, SIM, SMP, NC, IST). Three systems allowed for selective editing of CT or CBCT data with virtual tools and separate masking and display of structures, e.g. neighboring teeth (CDX, SIM,
NC). Two systems offered no individual tools for manual editing of three-dimensional models, but grey-value segmentation and separation of upper and lower jaw (SMP, IST).

Virtual models were displayed in 2D along crosssectional views and as 3D surface models. A transparent 3D display served for better visibility of underlying bone. A color display of intraoral scans was only possible with one system (IST) that provided a proprietary intraoral scanner (Table 5).

\section{CAD/CAM of drill guides}

Drill guides could either be designed by the software user (CDX, IST) or in a central service and production center. SIM and NC only allowed drill guide design through a central service and production center. One software system offered both an individual and a central service for design and production of drill guides (SMP). The tested software systems allowed tooth-, bone- or mucosa-supported (CDX, SIM, SMP) or tooth- or mucosa-supported (NC, IST) designs. Drill guides for fully guided implant placement could be designed and produced for 11 (CDX), 16 (SMP), 26 (SIM), 45 (IST) implant systems, respectively. One system only offered guided-implant placement for proprietary implants and pilot drill for third party implant types (NC) (Table 6).

The systems for an individual drill guide design (IST, CDX, SMP) allowed the user to define the bearing surface, the material thickness (IST, CDX), the tolerance between tooth surface and drill guide and the tolerance for inserting the drill sleeves, respectively (CDX). Undercut model surfaces were either virtually blocked out (CDX) or faded out (IST) automatically for control of intraoral seating. Drill sleeves were inserted according to the selected implant type. After defining the bearing surface, the software displayed a virtual model of the drill

Table 3 Assessment criteria for automatic and manual drill guide design and production

drill guide design and production supporting structures (teeth, bone, mucosa)

guiding protocol (guided pilot drill, guided drill sequence, guided implant placement) export of drill guide design data set

individual design and production of drill guide

central design and production of drill guide 

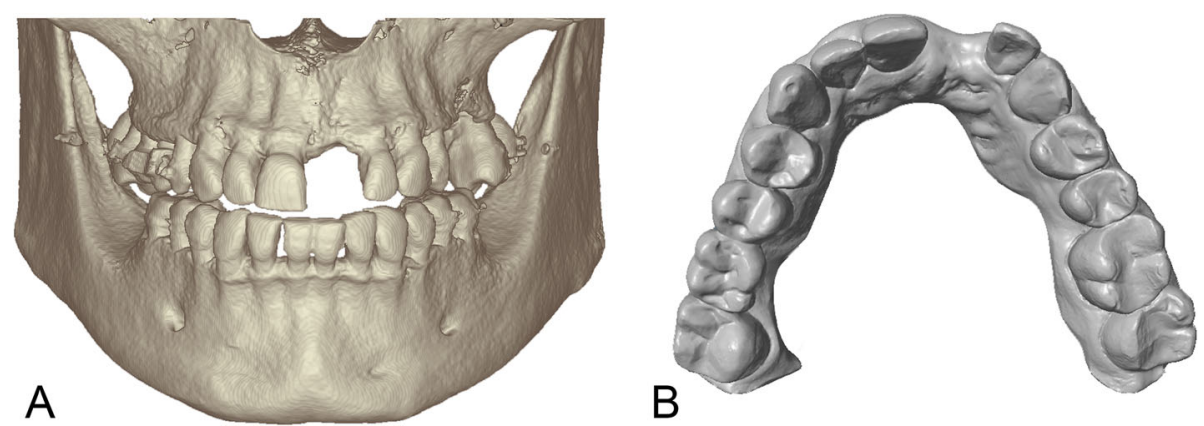

Fig. 1 Display of data import options. All systems could import (CB-)CT data displaying the alveolar bone in the implant region (a) and virtual stone casts representing tooth and mucosa surfaces (b)

guide (Fig. 2). The bearing surface is chosen differently in each system. With NC and SIM the operator is able to choose the extension by selecting the teeth. In IST and CDX the extension of the guide can be defined based on markers which define the border of the guide. This gives more flexibility to the operator. In SMP the bearing surface is chosen in only selected areas due to the "open design" of the final guide. In this virtual display, windows could be inserted for intraoperative seating control of the guide (CDX, IST). An extended software module for drill guide design integrated in one software required the user to get a special training (SMP). The drill guide was composed of supporting and connecting elements with various diameters resulting in a skeletonized contact surface. Compared to the designs where the guide covers the selected area of the tooth (closed design) the SMP guides follow an "open frame" design. The supporting areas on a tooth are selected and connected in a tube-like design.

Alternatively, to the individual design of drill guides, the system displayed a virtual preview that could not (SMP) or slightly be modified by the user (NC). In this case the drill guide design was forwarded to a production center or a specialized dental laboratory for design and manufacturing (SMP). Individual labeling with patient initials or identification code on the drill guide surface was possible in IST, CDX, SMP and exported in an STL-format for in-office manufacturing (CDX, SMP, IST) or sent to a production center (Table 4). Design and display of the drill guides in the different software systems are shown in Fig. 3.

Table 4 Data import options for all the tested systems CDX (coDiagnostiX, Dentalwings); NC (NobelClinician, Nobel Biocare); SIM (Simplant, Dentsply); SMP (Smop, Swissmeda) and IST (Implant Studio, 3Shape)

\begin{tabular}{|c|c|c|c|c|c|}
\hline & CDX & SIM & SMP & NC & IST \\
\hline \multicolumn{6}{|l|}{ (CB-)CT } \\
\hline DICOM & $\checkmark$ & $\checkmark$ & $\checkmark$ & $\checkmark$ & $\checkmark$ \\
\hline proprietary $\mathrm{CT}$ or $\mathrm{CBCT}$ scanner & $x$ & $x$ & $x$ & $x$ & from 2017 \\
\hline \multicolumn{6}{|l|}{ model scan } \\
\hline stl & $\checkmark$ & $\checkmark$ & $\checkmark$ & $\checkmark$ & $\checkmark$ \\
\hline other & $x$ & $x$ & .ply format & nxa format & dem format \\
\hline proprietary model scanners & $(\sqrt{ })$ & $x$ & $x$ & $\begin{array}{l}\text { Nobel } \\
\text { Procera G2 }\end{array}$ & $\checkmark$ \\
\hline \multicolumn{6}{|l|}{ intraoral scan } \\
\hline stl & $\checkmark$ & $\checkmark$ & $\checkmark$ & $x$ & $\checkmark$ \\
\hline other & $x$ & $x$ & $x$ & $x$ & $\begin{array}{l}. \mathrm{dcm} \text { format } \\
\text { with texture }\end{array}$ \\
\hline \multicolumn{6}{|l|}{ image registration } \\
\hline double scan protocol & $\checkmark$ & $\checkmark$ & $\checkmark$ & $\checkmark$ & $\checkmark$ \\
\hline single scan protocol with reference markers & $\checkmark$ & $\checkmark$ & $\checkmark$ & $\checkmark$ & $\checkmark$ \\
\hline single scan without reference markers & $\checkmark$ & $\checkmark$ & $\checkmark$ & $\checkmark$ & $\checkmark$ \\
\hline automatic registration & $x$ & $x$ & $x$ & $\checkmark$ & $x$ \\
\hline semi-automatic registration & $\checkmark$ & $\checkmark$ & $\checkmark$ & $\checkmark$ & $\checkmark$ \\
\hline
\end{tabular}


Table 5 Results for visualization of CT or CBCT and dental models in tested software systems. (*Bone density measurements are based on Hounsfield units used for CT data and are not valid for CBCT data)

\begin{tabular}{|c|c|c|c|c|c|}
\hline & CDX & SIM & SMP & NC & IST \\
\hline \multicolumn{6}{|l|}{ visualization of virtual stone casts } \\
\hline 2D display & $\checkmark$ & $\checkmark$ & $\checkmark$ & $\checkmark$ & $\checkmark$ \\
\hline 3D display & $\checkmark$ & $\checkmark$ & $\checkmark$ & $\checkmark$ & $\checkmark$ \\
\hline transparent display & $\checkmark$ & $\checkmark$ & $\checkmark$ & $\checkmark$ & $\checkmark$ \\
\hline color display & & & & & $\checkmark$ \\
\hline \multicolumn{6}{|l|}{ visualization of $\mathrm{CT}$ or $\mathrm{CBCT}$ data } \\
\hline orthopantomographic view & $\checkmark$ & $\checkmark$ & $\checkmark$ & $\checkmark$ & $\checkmark$ \\
\hline $2 \mathrm{D}$ cross-sectional images & $\begin{array}{l}\text { axial, } \\
\text { transversal, } \\
\text { tangential }\end{array}$ & $\begin{array}{l}\text { axial, } \\
\text { transversal, } \\
\text { tangential }\end{array}$ & $\begin{array}{l}\text { axial, } \\
\text { transversal, } \\
\text { tangential }\end{array}$ & $\begin{array}{l}\text { axial, } \\
\text { transversal, } \\
\text { tangential }\end{array}$ & $\begin{array}{l}\text { axial, } \\
\text { transversal, } \\
\text { tangential }\end{array}$ \\
\hline 3D model rendering & $\checkmark$ & $\checkmark$ & $\checkmark$ & $\checkmark$ & $\checkmark$ \\
\hline automatic and manual segmentation & $\checkmark$ & $\checkmark$ & $\checkmark$ & $\checkmark$ & $\checkmark$ \\
\hline Individual editing of imaging artifacts & $\checkmark$ & $\checkmark$ & $x$ & $\checkmark$ & $x$ \\
\hline tool for bone density measurement* & $\checkmark$ & $\checkmark$ & $x$ & $x$ & $\checkmark$ \\
\hline
\end{tabular}

\section{Discussion}

All tested implant planning systems used CT or CBCT DICOM data for bone diagnostics. None of the systems offered a proprietary CBCT scanner. To the knowledge of the authors, proprietary CBCT scanners are so far not available for any of the systems. Three-dimensional reconstructions and multiplanar cross-sections oriented along the alveolar process in the implant region were available in all systems to review important parameters for the implant position $[25,26]$.

With the clinical patient examples chosen in this study, imaging artefacts occurred distorting the tooth surface and bone volume. The examined software systems provided automatic segmentation of bone, teeth or soft tissues; however due to artifacts these default settings could not be used to display specific anatomical structures. Manual segmentation by limiting the window of grey values for the display of three-dimensional models was necessary and possible in all systems. Two systems did not offer tools to manually edit display of imaging data and two of the implant planning software provided tools for bone density measurement. Studies regarding grey values in CBCT data showed that they cannot be standardized and allocated to specific anatomical structures as in CT. Therefore, Hounsfield units used for interpretation of CT data are not applicable for CBCT data and bone density measurements in CBCT are not reliable [27].

The import, segmentation and pre-processing of radiographic data is crucial for the accurate transfer of the planned implant position to the surgical site. Radiographic data and virtual dental models are aligned with each other using either the tooth surface displayed both in CT or CBCT and in virtual dental models $[9,28]$ or with the help of reference markers in a radiographic splint $[11,12,15,29,30]$. Both workflows were available with the tested implant systems. Registration without a radiographic splint appears to be less time consuming as

Table 6 CAD/CAM options and supporting surfaces for implant drill guides produced with the tested software systems

\begin{tabular}{|c|c|c|c|c|c|}
\hline & CDX & SIM & SMP & NC & IST \\
\hline \multicolumn{6}{|l|}{ drill guide design (CAD) and production (CAM) } \\
\hline tooth support & $\checkmark$ & $\checkmark$ & $\checkmark$ & $\checkmark$ & $\checkmark$ \\
\hline bone support & $\checkmark$ & $\checkmark$ & $\checkmark$ & $x$ & $x$ \\
\hline mucosal support & $\checkmark$ & $\checkmark$ & $\checkmark$ & $\checkmark$ & $\checkmark$ \\
\hline fully guided drill and implant insertion & $\checkmark$ & $\checkmark$ & $\checkmark$ & $\begin{array}{l}\text { only } \\
\text { proprietary } \\
\text { implants }\end{array}$ & $\checkmark$ \\
\hline implant systems for fully guided drill & 11 & 26 & 16 & 1 & 45 \\
\hline export of drill guide design dataset for individual production & $\checkmark$ & $x$ & $\checkmark$ & $x$ & $\checkmark$ \\
\hline individual design of drill guide & $\checkmark$ & $x$ & $\checkmark$ & $x$ & $\checkmark$ \\
\hline central production of drill guide & $\checkmark$ & $\checkmark$ & $\checkmark$ & $\checkmark$ & $\checkmark$ \\
\hline
\end{tabular}




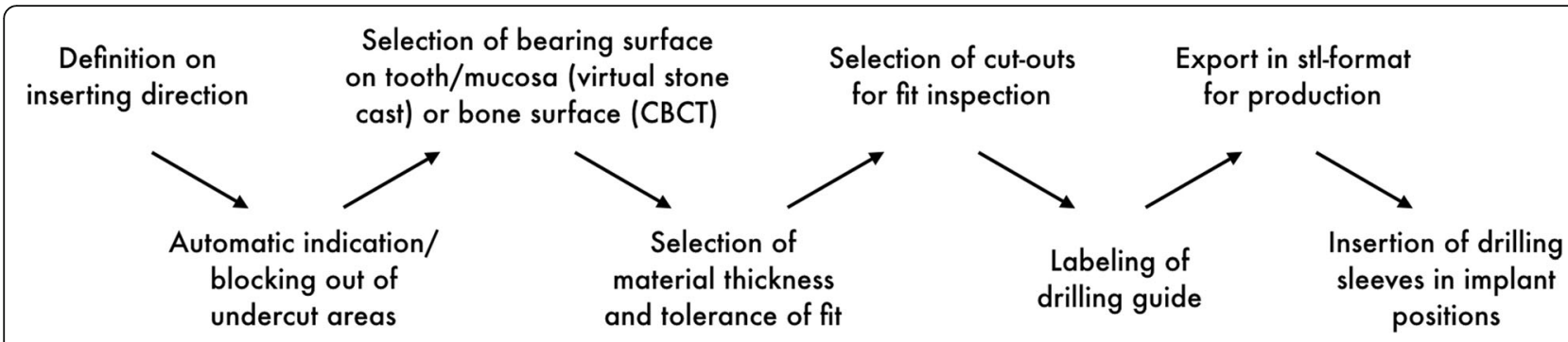

Fig. 2 Workflow for drill guide design with software systems for individual drill guide design (CDX, SMP, IST)

all examinations may be conducted without the preparation of a radiographic splint on a stone cast. However, misalignment between $\mathrm{CT}$ or CBCT and virtual models is known to occur after registration depending on the number of existing metal restorations [9].

The use of either an intraoral optical scan, or an impression or model scan, respectively, to produce a virtual dental model is freely selected by the user if the data is imported in the STL-format. One exception was found for $\mathrm{NC}$, only importing virtual models in a proprietary data format generated by a system-specific model scanner. Intraoral scans including information on the color of teeth and intraoral soft tissue (Trios, 3Shape) were only compatible for IST planning software. The use in a third-party software was only possible after export to an stl-format that does not contain texture information. Therefore, implant planning with consideration of tissue quality is hitherto only possible with one system that includes a proprietary intraoral scanner with texture information (IST).

Intraoral optical scanning reduces the steps and therefore time expenditure to obtain virtual models [31, 32]. Besides the promising efficiency of intraoral scans, the accuracy of intraoral optical scanning is still not validated in vivo. In contrast, extraoral optical scanning of stone casts showed high accuracy $(10 \mu \mathrm{m})$ [33]. However, the possible inaccuracy of a conventional intraoral impression and stone cast production are not included in the aforementioned studies. Inaccuracies of conventional intraoral impression should therefore be considered, when comparing the accuracy of intraoral optical impressions with extraoral model scans.

Depending on the used implant system either single steps or the full drill sequence and implant insertion is performed through the drill guide [10,34-36]. The examined software systems allowed guided implant placement for a various number of integrated systems except for one implant system (NC) that only offered guided implant placement for its proprietary implant system. The selection of implant systems for which guided implant placement was provided was restricted and did not correspond to the number of systems offered for visualization. The selection of an implant planning software is therefore dependent on the specific implant systems used in the daily routine.

The support of the drill guide on teeth and mucosa, respectively, allows a more accurate transfer of the implant position than bone support $[19,37,38]$. The user could choose between the three bearing surfaces with exception of two systems (NC, IST), where no bone support was possible. Furthermore, pins or provisional implants could be inserted with all systems to help the fixation of the drill guide during surgery as suggested previously by other authors $[39,40]$. Individual design of drill guides allowed the user to select bearing surfaces depending on each patient case. Whereas a closed guide design is suggested by most systems (NC, SIM, CDX, IST) an "open frame" design can be advantageous for more visibility, accessibility and less risk for interference with hard or soft tissue. Therefore, the insertion of windows in the closed design becomes important. With central design and production of drill guides, the user has to forward individual information regarding any specialties in the design prior to fabrication. The time consumption for personally designing and/or manufacturing of the drill guide and the cost of the software should be considered by the user, when using or choosing a virtual implant planning software. Two systems did not allow to individually plan nor individually fabricate the drill guides at the time of data collection (SIM, NC). To the knowledge of the authors, more recent versions of both software systems allowed individual production of the drill guide.

It has to be mentioned that user experience plays an important role in every CAD software. Depending on the user's experience, their affinity to digital products the learning curve can vary. In summary, the authors find one planning software more intuitive than the other, which is very subjective. Before chosing a system it is recommended to test as many as possible to find a satisfactory product.

\section{Conclusions}

Due to DICOM-interface, all implant systems could import radiographic data and three-dimensional reconstructions or two-dimensional cross-sections. When 


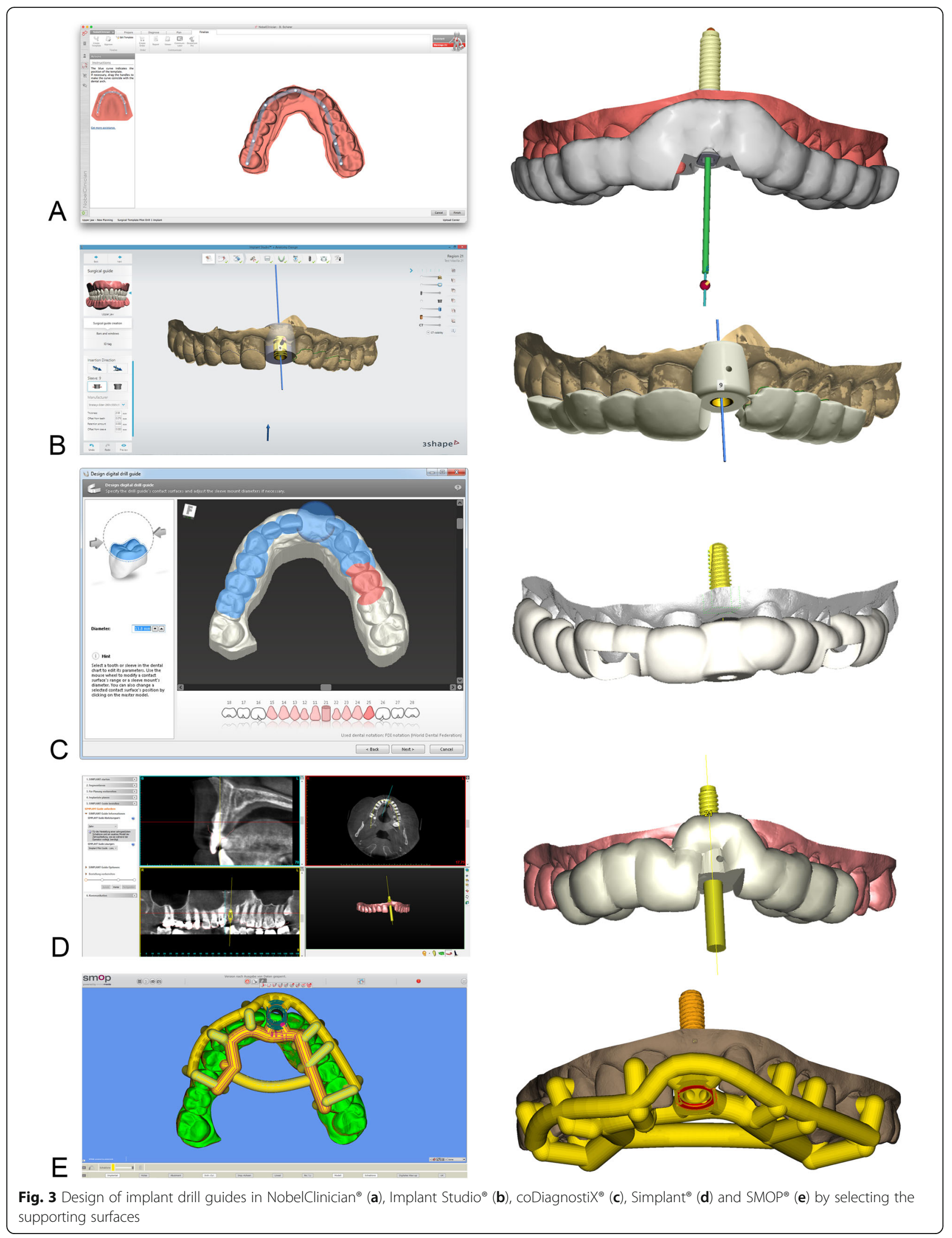


dental radiographic imaging is impaired by streaking artifacts, e.g. metallic restorations, all software systems allowed to manually segment the $\mathrm{CT}$ or $\mathrm{CBCT}$ data and CDX. SIM and NC offered a reduction of imaging artifacts by manual processing of data. Virtual implant planning systems allow either an individual design and production of drill guides or referral to a production facility where the fabrication is centralized. The construction of the drill guides result in similar designs except for the open design with SMP. Depending on the selected virtual planning software a varying selection of implant types may be planned for guided implant surgery. Further studies should investigate the time consumption associated with the use of the software to evaluate the relation of time and cost. Outsourcing parts of the planning process can become a viable option in the future and should be taken into consideration.

\section{Abbreviations}

CAD: Computer Aided Design; CAM: Computer Aided Manufacturing; CBCT: Cone beam computed tomography; CDX: coDiagnostiX; DICOM: Digital Imaging and Communication in Medicine; IST: Implant Studio; NC: NobelClinician; SIM: Simplant; SMP: Smop; STL: Standard Tesselation Language

\section{Acknowledgements}

Not applicable.

\section{Authors' contributions}

FK, JK and LW performed the data extraction, interpreted and analyzed the extracted data. DW and KN substantially contributed to the conception of the work, reviewed and edited the manuscript. TF conceived and designed the work and gave final approval of the version to be published. TF and FK wrote the manuscript with input from all authors. All authors read and approved the manuscript.

\section{Funding}

Not applicable. Open access funding provided by Projekt DEAL.

\section{Availability of data and materials}

The datasets generated and analysed during the current study are not publicly available since they are not encrypted but are available from the corresponding author on reasonable request.

\section{Ethics approval and consent to participate}

Not applicable.

\section{Consent for publication}

Not applicable.

\section{Competing interests}

The authors declare that they have no competing interests.

\section{Author details}

'Department of Oral and Maxillofacial Surgery, Translational Implantology, Medical Center - University of Freiburg, Faculty of Medicine, University of Freiburg, Hugstetter Str. 55, 79106 Freiburg, Germany. ${ }^{2}$ Department of Oral Implantology, Academisch Centrum Tandheelkunde Amsterdam (ACTA), Amsterdam, Netherlands. ${ }^{3}$ Charité - Universitätsmedizin Berlin, corporate member of Freie Universität Berlin, Humboldt-Universität zu Berlin, and Berlin Institute of Health, Department of Oral and Maxillofacial Surgery, Berlin, Germany.
Received: 31 March 2020 Accepted: 3 August 2020

Published online: 10 September 2020

\section{References}

1. Bornstein MM, Horner K, Jacobs R. Use of cone beam computed tomography in implant dentistry: current concepts, indications and limitations for clinical practice and research. Periodontol 2000. 2017;73(1): $51-72$.

2. Braut $\mathrm{V}$, Bornstein $\mathrm{MM}$, Kuchler $\mathrm{U}$, Buser $\mathrm{D}$. Bone dimensions in the posterior mandible: a retrospective radiographic study using cone beam computed tomography. Part 2--analysis of edentulous sites. Int I Periodontics Restorative Dent. 2014;34(5):639-47.

3. Ludlow JB, Ivanovic M. Comparative dosimetry of dental CBCT devices and 64-slice CT for oral and maxillofacial radiology. Oral Surg Oral Med Oral Pathol Oral Radiol Endod. 2008:106(1):106-14.

4. Schulze R, Bruellmann DD, Roeder F, d'Hoedt B. Determination of projection geometry from quantitative assessment of the distortion of spherical references in single-view projection radiography. Med Phys. 2004;31(10):2849-54.

5. Schulze R, Heil U, Gross D, Bruellmann DD, Dranischnikow E, Schwanecke U, Schoemer E. Artefacts in CBCT: a review. Dentomaxillofac Radiol. 2011;40(5): 265-73.

6. Zhao XZ, Xu WH, Tang ZH, Wu MJ, Zhu J, Chen S. Accuracy of computerguided implant surgery by a CAD/CAM and laser scanning technique. Chin J Dent Res. 2014:17(1):31-6.

7. Huotilainen E, Jaanimets R, Valášek J, Marcián P, Salmi M, Tuomi J, Mäkitie A, Wolff $J$. Inaccuracies in additive manufactured medical skull models caused by the DICOM to STL conversion process. J Craniomaxillofac Surg. 2014; 42(5):e259-65.

8. Maintz JB, Viergever MA. A survey of medical image registration. Med Image Anal. 1998;2(1):1-36

9. Flügge T, Derksen W, Te Poel J, Hassan B, Nelson K, Wismeijer D. Registration of cone beam computed tomography data and intraoral surface scans - a prerequisite for guided implant surgery with CAD/CAM drilling guides. Clin Oral Implants Res. 2017;28(9):1113-8.

10. Katsoulis J, Pazera P, Mericske-Stern R. Prosthetically driven, computerguided implant planning for the edentulous maxilla: a model study. Clin Implant Dent Relat Res. 2009:11(3):238-45.

11. Behneke A, Burwinkel M, Behneke N. Factors influencing transfer accuracy of cone beam CT-derived template-based implant placement. Clin Oral Implants Res. 2012;23(4):416-23.

12. Fortin $T$, Isidori $M$, Blanchet $E$, Perriat $M$, Bouchet $H$, Coudert JL. An imageguided system-drilled surgical template and trephine guide pin to make treatment of completely edentulous patients easier: a clinical report on immediate loading. Clin Implant Dent Relat Res. 2004:6(2):111-9.

13. Abbo B. Fixed complete denture using implants and computer-guided technology. Dent Today. 2009:28(6):88 90, 92-3.

14. Vercruyssen M, Coucke W, Naert I, Jacobs R, Teughels W, Quirynen M. Depth and lateral deviations in quided implant surgery: an RCT comparing guided surgery with mental navigation or the use of a pilot-drill template. Clin Oral Implants Res. 2015;26(11):1315-20.

15. Vercruyssen M, Fortin T, Widmann G, Jacobs R, Quirynen M. Different techniques of static/dynamic guided implant surgery: modalities and indications. Periodontol 2000. 2014;66(1):214-27.

16. Widmann G, Berggren JP, Fischer B, Pichler-Dennhardt AR, Schullian P, Bale $R$, Puelacher W. Accuracy of image-fusion Stereolithographic guides: mapping $C T$ data with three-dimensional optical surface scanning. Clin Implant Dent Relat Res. 2015;17(Suppl 2):e736-44.

17. Katsoulis J, Enkling N, Takeichi T, Urban IA, Mericske-Stern R, Avrampou M. Relative bone width of the edentulous maxillary ridge. Clinical implications of digital assessment in presurgical implant planning. Clin Implant Dent Relat Res. 2012;14(Suppl 1):e213-23.

18. Turbush SK, Turkyilmaz I. Accuracy of three different types of stereolithographic surgical guide in implant placement: an in vitro study. J Prosthet Dent. 2012:108(3):181-8.

19. Raico Gallardo YN, da Silva-Olivio IRT, Mukai E, Morimoto S, Sesma N, Cordaro L. Accuracy comparison of guided surgery for dental implants according to the tissue of support: a systematic review and meta-analysis. Clin Oral Implants Res. 2017:28(5):602-12.

20. Cassetta M, Di Mambro A, Giansanti M, Stefanelli LV, Cavallini C. The intrinsic error of a stereolithographic surgical template in implant guided surgery. Int J Oral Maxillofac Surg. 2013;42(2):264-75. 
21. Tahmaseb A, Wu V, Wismeijer D, Coucke W, Evans C. The accuracy of static computer-aided implant surgery: a systematic review and meta-analysis. Clin Oral Implants Res. 2018;29(Suppl 16):416-35.

22. Neugebauer J, Kistler F, Kistler S, Züdorf G, Freyer D, Ritter L, Dreiseidler T, Kusch J, Zöller JE. CAD/CAM-produced surgical guides: optimizing the treatment workflow. Int J Comput Dent. 2011;14(2):93-103.

23. Bindl A. Clinical application of fully digital Cerec surgical guides made inhouse. Int J Comput Dent. 2015;18(2):163-75.

24. Sarment DP, Sukovic P, Clinthorne N. Accuracy of implant placement with a stereolithographic surgical guide. Int J Oral Maxillofac Implants. 2003;18(4): $571-7$.

25. Schwarz MS, Rothman SL, Rhodes ML, Chafetz N. Computed tomography: part I. preoperative assessment of the mandible for endosseous implant surgery. Int J Oral Maxillofac Implants. 1987;2(3):137-41.

26. Hatcher DC, Dial C, Mayorga C. Cone beam CT for pre-surgical assessment of implant sites. J Calif Dent Assoc. 2003;31(11):825-33.

27. Pauwels R, Jacobs R, Singer SR, Mupparapu M. CBCT-based bone quality assessment: are Hounsfield units applicable? Dentomaxillofac Radiol. 2015; 44(1):20140238.

28. Joda T, Gallucci GO. The virtual patient in dental medicine. Clin Oral Implants Res. 2015;26(6):725-6.

29. Birkfellner W, Solar P, Gahleitner A, Huber K, Kainberger F, Kettenbach J, Homolka P, Diemling M, Watzek G, Bergmann H. In-vitro assessment of a registration protocol for image guided implant dentistry. Clin Oral Implants Res. 2001;12(1):69-78.

30. Swennen GR, Barth EL, Eulzer C, Schutyser F. The use of a new 3D splint and double $C T$ scan procedure to obtain an accurate anatomic virtual augmented model of the skull. Int J Oral Maxillofac Surg. 2007;36(2):146-52 Epub 2007 Jan 8.

31. Flügge TV, Schlager S, Nelson K, Nahles S, Metzger MC. Precision of intraoral digital dental impressions with iTero and extraoral digitization with the iTero and a model scanner. Am J Orthod Dentofac Orthop. 2013;144(3):4718

32. Patzelt SB, Emmanouilidi A, Stampf S, Strub JR, Att W. Accuracy of full-arch scans using intraoral scanners. Clin Oral Investig. 2014;18(6):1687-94.

33. Persson A, Andersson M, Oden A, Sandborgh-Englund G. A threedimensional evaluation of a laser scanner and a touch-probe scanner. J Prosthet Dent. 2006;95(3):194-200.

34. Lal K, White GS, Morea DN, Wright RF. Use of stereolithographic templates for surgical and prosthodontic implant planning and placement. Part I The concept. J Prosthodont. 2006;15(1):51-8.

35. Lal K, White GS, Morea DN, Wright RF. Use of stereolithographic templates for surgical and prosthodontic implant planning and placement. Part II. A clinical report. J Prosthodont. 2006:15(2):117-22.

36. Jung RE, Schneider D, Ganeles J, Wismeijer D, Zwahlen M, Hämmerle CH, Tahmaseb A. Computer technology applications in surgical implant dentistry: a systematic review. Int J Oral Maxillofac Implants. 2009;24(Suppl): 92-109.

37. Van Assche N, Vercruyssen M, Coucke W, Teughels W, Jacobs R, Quirynen M. Accuracy of computer-aided implant placement. Clin Oral Implants Res. 2012;23(Suppl 6):112-23.

38. Tahmaseb A, Wismeijer D, Coucke W, Derksen W. Computer technology applications in surgical implant dentistry: a systematic review. Int J Oral Maxillofac Implants. 2014;29(Suppl):25.

39. Arisan V, Karabuda ZC, Ozdemir T. Accuracy of two stereolithographic guide systems for computer-aided implant placement: a computed tomographybased clinical comparative study. J Periodontol. 2010;81(1):43-51.

40. D'Haese J, Van De Velde T, Elaut L, De Bruyn H. A prospective study on the accuracy of mucosally supported stereolithographic surgical guides in fully edentulous maxillae. Clin Implant Dent Relat Res. 2012;14(2):293-303.

\section{Publisher's Note}

Springer Nature remains neutral with regard to jurisdictional claims in published maps and institutional affiliations.

\section{Ready to submit your research? Choose BMC and benefit from}

- fast, convenient online submission

- thorough peer review by experienced researchers in your field

- rapid publication on acceptance

- support for research data, including large and complex data types

- gold Open Access which fosters wider collaboration and increased citations

- maximum visibility for your research: over $100 \mathrm{M}$ website views per year

At $\mathrm{BMC}$, research is always in progress.

Learn more biomedcentral.com/submissions 